\title{
What do we know about Last Interglacial sea level?
}

\author{
Andrea Dutton ${ }^{1}$ and Natasha L.M. Barlow ${ }^{2}$
}

\begin{abstract}
The Last Interglacial (LIG, Marine Isotope Stage 5e) represents the most recent time period when the polar ice sheets retreated significantly from their present extent, and hence has frequently been cited as a useful, though imperfect, analogue to understand future ice-sheet and sea-level response to a warming climate.
\end{abstract}

The LIG sea-level highstand that persisted from $\sim 129$ to 116 thousand years (kyr) ago has long been acknowledged to be higher than present sea level, though the magnitude of peak sea level and the stability of sea level during the highstand are still actively debated (Dutton et al. 2015a; Austermann et al. 2017; Barlow et al. 2018). While there are important differences between modern, anthropogenically-driven global warming and the orbitally driven warmth of the LIG, there are several relevant and valuable observations to be made. Among these are (1) understanding the sensitivities of the ice sheets in Greenland and Antarctic to warming; (2) identifying the mechanisms contributing to the millennial-scale evolution of climate and sea level during the interglacial; and (3) mapping out the sea-level budget, including the sources, magnitudes, and timing of meltwater contributions. Fundamentally, one of the most critical questions to answer is whether there was a significant contribution from the Antarctic ice sheet to LIG sea level, which is still uncertain.

\section{Magnitude of peak LIG sea level}

Sea level varies geographically, due to a combination of processes including glacial isostatic adjustment (GIA) and dynamic topography (Milne et al. this issue; Austermann and Forte, this issue). Therefore, the maximum height of LIG sea level will not be the same at every location or occur at the same time (Dutton and Lambeck 2012). Several research groups have independently assessed peak global mean sea level (GMSL) from a combination of individual observations of relative sea level from globally distributed localities (Kopp et al. 2009; Dutton and Lambeck 2012; Düsterhus et al. 2016) (Fig. 1). Though these estimates vary somewhat from each other, GIA-corrected reconstructions for GMSL appear to converge on values of 6-9 $\mathrm{m}$ above present for the peak of LIG sea-level rise. An important qualification to this interpreted range is the recognition that vertical motion of the earth's surface due to mantle convection (dynamic topography) since the LIG could easily be on the order of a few meters - up or down - depending on the location (Austermann et al. 2017). While this casts additional uncertainty on the provisional $\sim 6-9$ m estimate, it is not straightforward to provide either accurate or precise assessments of the magnitude of the dynamic topography signal for specific sites, and hence difficult to determine if and how this estimate of peak sea level should be further modified.

\section{Sources of LIG sea-level rise}

There is evidence from both observations and models that the Greenland ice sheet was significantly smaller than present during the LIG. Though the summary of several studies presented by Dutton et al. (2015a) indicated a convergence towards $2 \mathrm{~m}( \pm 1.5)$ of GMSL contribution from Greenland, a subsequent modeling study increased this estimate to $5.1 \mathrm{~m} \mathrm{(4.1}$ to $6.2,95 \%$ credible interval; Yau et al. 2016). To some extent, the disagreement between studies in terms of inferred icesheet size may be due to the conflicting implications of total gas content in the ice cores versus temperature estimates. Nonetheless, ice-sheet models consistently produce a couple meters or more of sea-level rise coming from Greenland, with peak contributions at $121-122 \mathrm{kyr}$ before present. In the absence of near-field data for Antarctica, estimates for an Antarctic contribution to LIG GMSL have typically been calculated by subtracting the contributions of other sources (Greenland, thermal expansion, and mountain glaciers) from far-field sea-level estimates. Given the large unknowns that exist in both peak GMSL and the Greenland

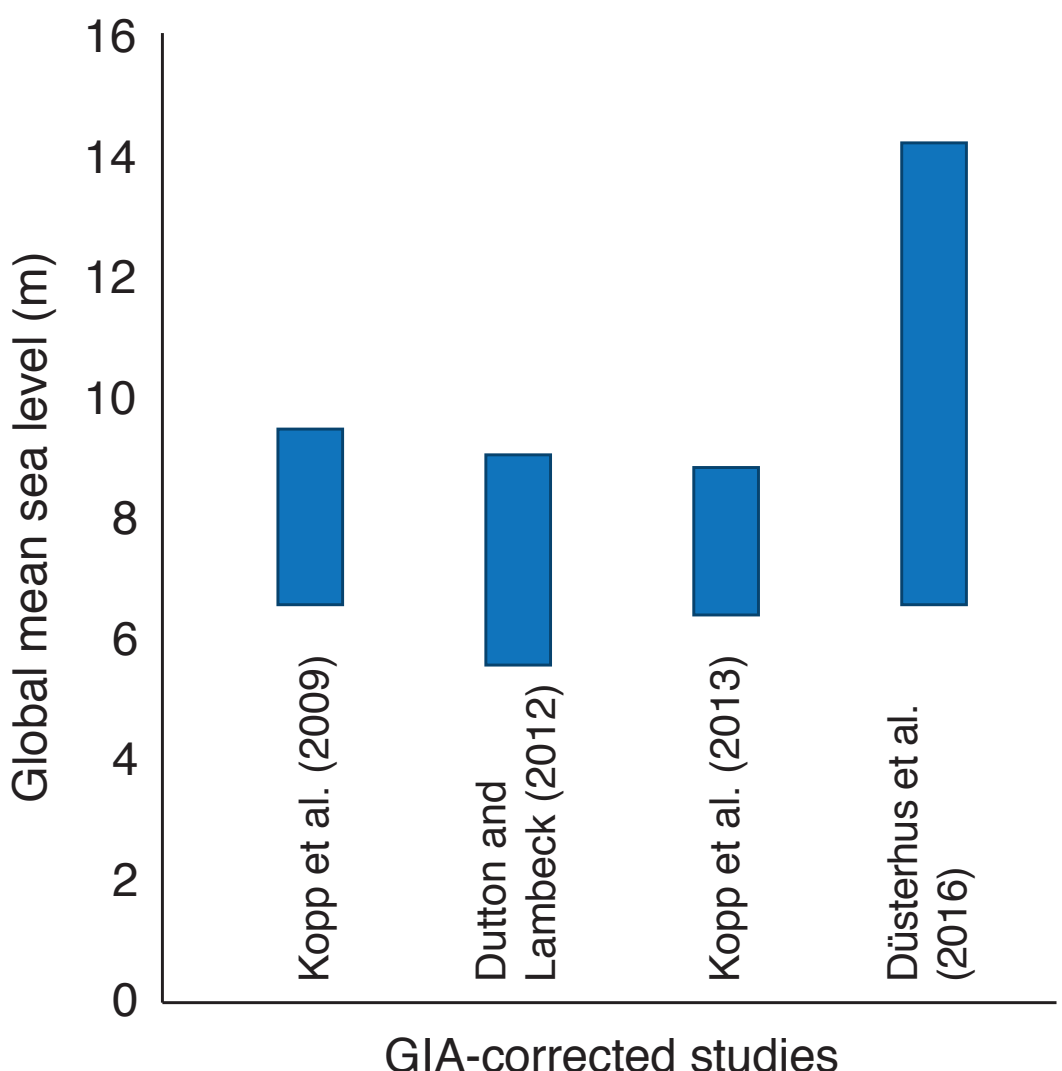

Figure 1: Estimates of global mean sea-level highstand during the Last Interglacial compared to today. All studies correct for glacial isostatic adjustment. ice-sheet contribution, this approach does not allow us to definitively determine whether a sector of the Antarctic ice sheet collapsed during the LIG. However, based on sediment provenance from circum-Antarctic cores, or estimates of sufficiently high GMSL early in the LIG (prior to significant retreat in Greenland), some have argued for a smaller Antarctic ice sheet compared to present at some point during the LIG (Dutton et al. 2015b; Wilson et al. 2018). Confidently "fingerprinting" a few meters of GMSL contribution from either Greenland or Antarctica by using the expected patterns in spatial variability of relative sea level due to GIA to identify the timing and sources of meltwater contribution is a provocative idea. However, this approach would require decimeter-scale vertical precision, which is typically well within the uncertainties of geologic sea-level indicators, hence making the fingerprint of individual ice sheets difficult to carry out in practice.

\section{Evolution of the sea-level highstand}

The sea-level highstand has been variously reported to have had anywhere from one 


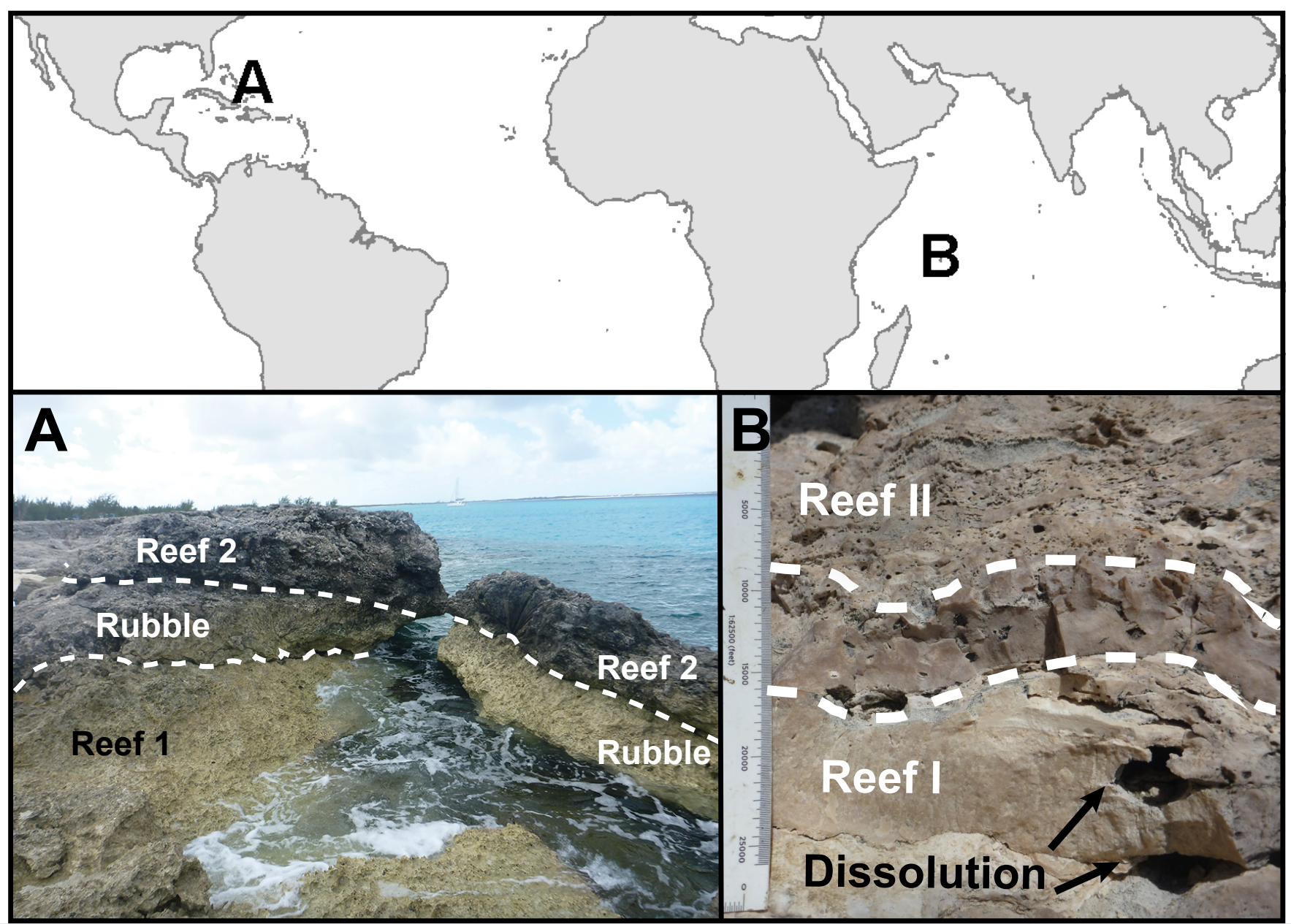

Figure 2: Field evidence from LIG sequences in (A) The Bahamas (Skrivanek et al. 2018) and (B) Seychelles (Vyverberg et al. 2018) that is consistent with an ephemeral sealevel fall occurring between the development of stratigraphically distinct reef units. Depending on the site, truncated corals in the lower reef, extensive dissolution on the reef surface, or sequence of freshwater and marine cements provide evidence of an ephemeral sea-level fall.

to four peaks, where multiple peaks define millennial-scale fluctuations in sea level. Some reconstructions simply connect ageelevation data points from corals with a line, which ignores potential changes in coral paleowater depths and contextual sedimentary and stratigraphic information from fossil reef outcrops. While the coral age-elevation-taxonomy compilation by Hibbert et al. (2016) drives the point home that paleowater depth ranges of corals can be large, this does not render the entire fossil coral archive useless for addressing the question of whether there were sea-level oscillations during the LIG. Instead, sedimentary evidence for changes in water depth, including intervals of subaerial exposure within the fossil reef or evidence of rapid reef accretion, can be used to more confidently interpret possible meter-scale changes. In fact, several studies have pointed to compelling evidence for abrupt changes in sea level preserved in LIG reef sequences (e.g. Blanchon et al. 2009; Skrivanek et al. 2018). The magnitude of these changes is poorly constrained, however. To confidently confirm these rapid sea-level changes at a global scale, more evidence needs to be amassed from geographically diverse sites with robust chronologies. Some recent studies have argued that a sea-level fall of $>4 \mathrm{~m}$, as indicated by Kopp et al. (2009), is not plausible given constraints from phreatic overgrowths in Mallorca caves (Polyak et al. 2018) and other lines of observational and modeling evidence (Barlow et al. 2018). However, these studies do not preclude the possibility of smaller, meter-scale sea-level fluctuation(s) in the low latitude reef sites during the LIG (Fig. 2).

\section{Implications for links between} climate, ice sheets, and sea level

There is great interest within the community to move beyond the big-picture view of the correspondence between peak temperatures and peak GMSL during the LIG, for example as depicted in Dutton et al. (2015a). Increasing attention is being drawn to millennial-scale changes in temperature, ice-sheet response, and the accompanying changes in sea level during the LIG to understand the thresholds for ice-sheet collapse, the temporal relation between climate forcing and sea level, and the rates of sea-level change. One of the biggest challenges in linking climate signals to observations of sea-level change is developing accurate, precise, and highly resolved chronologies that can confidently be correlated to other records, for instance, between data from deep-sea cores and U-Th dated archives of sea-level change in coral reefs. Tzedakis et al. (2018) demonstrate that climate in the North Atlantic region was more variable during the LIG than the Holocene, but it is yet to be firmly established how that variability links to concomitant changes in ice volume, meltwater input and sea level.

What is clear, however, is that the more we can extract from the paleo record about the phasing, magnitude, and sources of meltwater input into the oceans, the better we can constrain the models that are used to project future sea-level rise. We envision that interdisciplinary approaches to this question will be essential to unravelling the dynamics of shrinking polar ice sheets in a warming world.

\section{AFFILIATIONS}

'Department of Geological Sciences, University of Florida, Gainesville, USA

${ }^{2}$ School of Earth and Environment, University of Leeds, UK

\section{CONTACT}

Andrea Dutton: adutton@ufl.edu

\section{REFERENCES}

Austermann J et al. (2017) Sci Adv 3: e1700457

Barlow NLM et al. (2018) Nat Geosci 11: 627-634 Blanchon P et al. (2009) Nature 458: 881-884 Düsterhus A et al. (2016) Geophys J Int 206: 900-920 Dutton A, Lambeck K (2012) Science 337: 216-219 Dutton A et al. (2015a) Science 349: aaa4019 Dutton A et al. (2015b) Quat Sci Rev 107: 182-196 Hibbert FD et al. (2016) Quat Sci Rev 145: 1-56 Kopp RE et al. (2009) Nature 462: 863-867 Polyak VJ et al. (2018) Nat Geosci 11: 860-864 Skrivanek A et al. (2018) Quat Sci Rev 200: 160-177 Tzedakis PC et al. (2018) Nat Commun 9: 4235 Vyverberg Ket al. (2018) Mar Geol 399: 170-187 Wilson DJ et al. (2018) Nature 561: 383-386 Yau AM et al. (2016) Proc Natl Acad Sci 113: 9710-9715 